VoL. 52 (1995) [41-61]

\title{
ON GROUP UNIFORMITIES ON SQUARE OF A SPACE AND EXTENDING PSEUDOMETRICS II
}

\author{
Michael G. TKaČenko
}

\begin{abstract}
We find topological conditions on a space $X$ under which the left (right, or twosided) uniformity of the free topological group $F(X)$ induces the universal uniformity $U_{X^{2}}$ or the product uniformity $\mathcal{U}_{X} \times \mathcal{U}_{X}$ on the square of $X$. Special attention is given to $k_{\omega}$-spaces and metrisable spaces. The main technical tool in the paper is an extension of certain continuous pseudometrics from $X^{2}$ to $F(X)$ considered by the author in the previous volume of this journal.
\end{abstract}

\section{Introduction}

By a theorem of Graev [5], any continuous pseudometric $d$ on a Tikhonov space $X$ extends to an invariant pseudometric $\hat{d}$ on the free topological group $F(X)$. This result was applied by Pestov [9] to prove the equality $\left.{ }^{*} \mathcal{V}^{*}\right|_{X}=\mathcal{U}_{X}$ for every Tikhonov space $X$, where ${ }^{*} \mathcal{V}^{*}$ is the two-sided uniformity of $F(X)$ and $\mathcal{U}_{X}$ is the universal uniformity of $X$ (that is, the finest uniformity on $X$ compatible with the topology of $X$ ). A generalisation of the above equality for uniform free topological groups was obtained by Nummela [8].

Our aim is to study the uniformities on $X^{2}$ induced by ${ }^{*} \mathcal{V}, \mathcal{V}^{*}$ and ${ }^{*} \mathcal{V}^{*}$, the left, right and two-sided group uniformities of $F(X)$. In talking about induced uniformities on $X^{2}$, it is understood that we identify $X^{2}$ with a subspace of $F(X)$ under the embedding $(x, y) \mapsto x \cdot y ; x, y \in X$. So, we can formulate the following three problems (see [17]).

Problem A. What are the relations between $\left.{ }^{*} \mathcal{V}\right|_{X^{2}},\left.\mathcal{V}^{*}\right|_{X^{2}}$ and $\left.{ }^{*} \mathcal{V}^{*}\right|_{X^{2}}$ on the one hand and $\mathcal{U}_{X} \times \mathcal{U}_{X}, \mathcal{U}_{X^{2}}$ on the other $\left(\mathcal{U}_{X^{2}}\right.$ stands for the universal uniformity on $\left.X^{2}\right)$ ?

This general problem can be specialised as follows.

Problem B. When does the equality $\left.{ }^{*} \mathcal{V}^{*}\right|_{X^{2}}=\mathcal{U}_{X} \times \mathcal{U}_{X}$ hold?

Problem C. For which spaces $X$ does the equality $\left.{ }^{*} \mathcal{V}^{*}\right|_{X^{2}}=\mathcal{U}_{X^{2}}$ hold?

Received 7th September, 1994

Copyright Clearance Centre, Inc. Serial-fee code: 0004-9729/95 \$A2.00+0.00. 
One can as well replace ${ }^{*} \mathcal{V}^{*}$ by ${ }^{*} \mathcal{V}$ or $\mathcal{V}^{*}$ in Problems $\mathrm{B}$ and $\mathrm{C}$, thus obtaining four more problems. The resulting problems will be denoted by the same letters. The majority of these problems is solved here by means of a simultaneous extension method that applies to certain pairs $\left(d_{1}, d_{2}\right)$ of continuous pseudometrics $d_{1}$ and $d_{2}$ on $X$ and $X^{2}$ respectively and produces continuous seminorms on $F(X)$ (see Theorems 1.4, 2.1 and 3.1 of $[17])$.

We start with general assertions about uniformities on topological groups. Then we show that both uniformities $\left.{ }^{*} \mathcal{V}\right|_{X^{2}}$ and $\left.\mathcal{V}^{*}\right|_{X^{2}}$ are finer than $\mathcal{U}_{X} \times \mathcal{U}_{X}$ (Theorem 1.6), that contributes to Problem A. As an application of simple topological tools we prove that the equality $\left.{ }^{*} \mathcal{V}^{*}\right|_{X^{2}}=\mathcal{U}_{X} \times \mathcal{U}_{X}$ holds for every pseudocompact space $X$ (Theorem 1.8), thus giving a partial answer to Problem B. A complete solution of Problem B is given in Theorem 2.1: the equality $\left.{ }^{*} \mathcal{V}^{*}\right|_{X^{2}}=\mathcal{U}_{X} \times \mathcal{U}_{X}$ holds if and only if there exists an infinite cardinal $\tau$ such that $X$ is pseudo- $\tau$-compact and a $P_{\tau}$-space simultanuously. This characterisation remains valid if one replaces ${ }^{*} \mathcal{V}^{*}$ by ${ }^{*} \mathcal{V}$ or $\mathcal{V}^{*}$.

Problem $\mathrm{C}$ seems the most difficult among the others. First, we characterise spaces $X$ satisfying the condition $\left.{ }^{*} \mathcal{V}\right|_{X^{2}}=\mathcal{U}_{X^{2}}$ (or equivalently, $\left.\mathcal{V}^{*}\right|_{X^{2}}=\mathcal{U}_{X^{2}}$ ): if $X$ is not a $P$-space then the above condition is equivalent to the requirement that the projection $p: X^{2} \rightarrow X$ is $z$-closed, and for a $P$-space $X$ it is equivalent to the condition that for every open cover $\gamma$ of $X^{2}$ there exists a disjoint open cover $\mu$ of $X$ such that $\mu \times \mu=\{U \times V: U, V \in \mu\}$ is finer than $\gamma$ (Theorem 3.1).

We also show that the equality $\left.{ }^{*} \mathcal{V}^{*}\right|_{X^{2}}=\mathcal{U}_{X^{2}}$ holds for each $k_{\omega}$-space $X$ (Theorem 4.2) and characterise metrisable spaces satisfying it (Theorem 4.4): the criterion is that a metrisable space must be locally compact or the set $X^{\prime}$ of all non-isolated points of $X$ must be compact.

All spaces considered are assumed completely regular. We say that $X$ is a $P$-space if every $G_{\delta}$-set in $X$ is open. A space $X$ is said to be pseudo- $\tau$-compact if every locally finite family of open sets in $X$ has cardinality strictly less than $\tau$. The Cech-Stone compactification of a space $X$ is denoted by $\beta X$.

The set of positive integers is denoted by $N^{+} ; \mathbf{R}$ stands for the reals with the interval topology.

Every element $g$ of the free topological group $F(X)$ on a space $X$ has the form $x_{1}^{e_{1}} \cdot \ldots \cdot x_{n}^{e_{n}}$ for some $x_{1}, \ldots, x_{n} \in X$ and $\varepsilon_{1}, \ldots, \varepsilon_{n}= \pm 1$. We put $l_{+}(g)=\{i \leqslant n$ : $\left.\varepsilon_{i}=1\right\}$ and $l_{-}(g)=\left\{i \leqslant n: \varepsilon_{i}=-1\right\}$. Then we define a subgroup $G(X)$ of $F(X)$ by

$$
G(X)=\left\{g \in F(X): l_{+}(g)=l_{-}(g)\right\}
$$

Note that $G(X)$ is an open subgroup of $F(X)[17]$.

All the necessary facts in uniform space theory are contained in [2, Chapter 8] or [6, Chapters 1-3]. An exposition of results on uniform structures on topological groups 
is given in $[\mathbf{1 0}]$.

\section{Preliminary facts and Results}

Let $G$ be a topological group with identity $e$ and $O$ a neighbourhood of $e$ in $G$. We put

$$
U_{O}^{l}=\left\{(g, h) \in G \times G: g^{-1} \cdot h \in O\right\}, \quad U_{O}^{r}=\left\{(g, h) \in G \times G: g \cdot h^{-1} \in O\right\},
$$

and $U_{O}=U_{O}^{l} \cap U_{O}^{r}$. Recall that a base of the left (right, two-sided) uniformity ${ }^{*} \mathcal{V}_{G}$ (respectively $\mathcal{V}_{G}^{*},{ }^{*} \mathcal{V}_{G}^{*}$ ) of the group $G$ consists of the sets $U_{O}^{t}$ (respectively $U_{O}^{r}, U_{O}$ ) where $O$ runs through all neighbourhoods of $e$ in $G$.

The following notion seems to be folklore.

Definition 1.1: Let $\tau$ be an infinite cardinal. A subset $Y \subseteq X$ is said to be $\tau$-bounded in a uniform space $(X, \mathcal{U})$ if for each $U \in \mathcal{U}$ there exists a subset $A \subseteq X$, $|A| \leqslant \tau$, such that $Y \subseteq \bigcup_{a \in A} B(a, U)$, where $B(a, U)=\{x \in X:(a, x) \in U\}$.

If one puts $Y=X$ in Definition 1.1, the notion of a $\tau$-bounded uniform space $(X, \mathcal{U})$ will be obtained. For the sake of completeness we present a proof of the following wellknown result. Recall that $\mathcal{U}_{X}$ always stands for the universal uniformity of a space $X$.

Assertion 1.2. A uniform space $(X, \mathcal{U})$ is $\tau$-bounded if and only if the space $X$ is pseudo- $\tau^{+}$-compact.

Proof: The necessity. Suppose that there exists a locally finite family $\gamma$ of open sets in $X,|\gamma|=\tau^{+}$. For every $V \in \gamma$ define a continuous real-valued function $f_{V}$ on $X, 0 \leqslant f_{V} \leqslant 1$, such that $f_{V}$ is equal to 1 at some point of $V$ and vanishes outside of $V$. Put

$$
d(x, y)=\sum_{V \in \gamma}\left|f_{V}(x)-f_{V}(y)\right| ; \quad x, y \in X .
$$

Then $d$ is a continuous pseudometric on $X$. The set $W=\left\{(x, y) \in X^{2}: d(x, y)<1\right\}$ is an open entourage of the diagonal in $X^{2}$ and $W \in \mathcal{U}_{X}$. It is easy to verify that no subset $A \subseteq X$ with $|A| \leqslant \tau$ satisfies the condition $X=\bigcup_{a \in A} B(a, W)$ of Definition 1.1, that is, the uniform space $\left(X, \mathcal{U}_{X}\right)$ is not $\tau$-bounded.

Sufficiency. Suppose that $\left(X, \mathcal{U}_{X}\right)$ is not $\tau$-bounded and choose an element $W \in$ $\mathcal{U}_{X}$ witnessing that. By Corollary 8.1.11 of [2] there exists a continuous pseudometric $\varrho$ on $X$ such that $\left\{(x, y) \in X^{2}: \varrho(x, y)<1\right\} \subseteq W$. Let $K$ be a maximal subset of $X$ with the property that $\varrho(a, b) \geqslant 1$ for all distinct $a, b \in K$. Then $|K|>\tau$ by the choice of $W$ and $\varrho$. Obviously, the family of all balls of radius $1 / 3$ with points of $K$ as centers is discrete (hence locally finite) and has cardinality greater than $\tau$. 
DEFINITION 1.3: A subset $Y$ of a topological group $G$ is called left- $\tau$-bounded in $G$ if for every neighbourhood $V$ of the identity in $G$ there exists a subset $A \subseteq G$ such that $|A| \leqslant \tau$ and $Y \subseteq A \cdot V$; analogously, the inclusion $Y \subseteq V \cdot A$ defines the notion of right- $\tau$-boundedness in $G$.

If a subset $Y$ of $G$ is left- and right- $\tau$-bounded in $G$, we shall simply say that $Y$ is $\tau$-bounded in $G$.

Note that $Y$ is left- $\tau$-bounded (right- $\tau$-bounded) in $G$ if and only if $Y$ is a $\tau$ bounded subset of $\left(G,{ }^{*} \mathcal{V}\right)$ (respectively $\left(G, \mathcal{V}^{*}\right)$ ). We also mention that the subset $A$ of $G$ in Definition 1.3 can be chosen to satisfy the condition $A \subseteq Y$.

Assertion 1.4. If $Y$ is (right-) left- $\tau$-bounded in a topological group $G$ then $Y \cdot Y$ is (right-) left- $\tau$-bounded in $G$.

Proof: It suffices to consider the "left" case. Let $V$ and $V_{1}$ be neighbourhoods of the identity in $G, V_{1}^{2} \subseteq V$. There exists a subset $B$ of $G,|B| \leqslant \tau$, such that $Y \subseteq B \cdot V_{1}$. For each $b \in B$ choose a neighbourhood $W_{b}$ of the identity satisfying the condition $b^{-1} \cdot W_{b} \cdot b \subseteq V_{1}$ and find a subset $C_{b}$ of $G$ of cardinality $\leqslant \tau$ with $Y \subseteq C_{b} \cdot W_{b}$. Put $C=\bigcup_{b \in B} C_{b}$ and $A=C \cdot B$. Obviously, $|A| \leqslant|C| \leqslant \tau$. We claim that $Y \cdot Y \subseteq A \cdot V$. Indeed, let $x, y \in Y$ be arbitrary. Then $y \in b \cdot V_{1}$ for some $b \in B$. Since $Y \subseteq C_{b} \cdot W_{b}$, there exists $c \in C_{b}$ such that $x \in c \cdot W_{b}$. We have

$$
x \cdot y \in c \cdot W_{b} \cdot b \cdot V_{1}=c \cdot b \cdot\left(b^{-1} \cdot W_{b} \cdot b\right) \cdot V_{1} \subseteq c \cdot b \cdot V_{1}^{2} \subseteq c \cdot b \cdot V,
$$

where $c \cdot b \in C \cdot B=A$. Thus, $Y \cdot Y \subseteq A \cdot V$.

Assertion 1.5. Suppose that $Y$ is a $\tau$-bounded set in a topological group $G$ with the two-sided uniformity ${ }^{*} \mathcal{V}^{*}$. Then $Y$ is a $\tau$-bounded subset of $\left(G,{ }^{*} \mathcal{V}^{*}\right)$.

Proof: Let $V$ be a neighbourhood of the identity in $G$. It suffices to define a subset $A \subseteq G$ such that $|A| \leqslant \tau$ and $Y \subseteq \bigcup\{a \cdot V \cap V \cdot a: a \in A\}$. Choose a symmetric neighbourhood $V_{1}$ of the identity so that $V_{1}^{3} \subseteq V$ and let the subset $B$ of $G$ satisfy $Y \subseteq B \cdot V_{1},|B| \leqslant \tau$. For every $b \in B$ find a neighbourhood $W_{b}$ of the identity such that $W_{b} \subseteq V$ and $b^{-1} \cdot W_{b} \cdot b \subseteq V_{1}$. For each $b \in B$ there exists a subset $A_{b} \subseteq G$ such that $Y \subseteq W_{b} \cdot A_{b}$ and $\left|A_{b}\right| \leqslant \tau$. We claim that the set $A=\bigcup_{b \in B} A_{b}$ works. Indeed, let $y \in Y$ be arbitrary. Then $y \in b \cdot V_{1}$ for some $b \in B$ and $y \in W_{b} \cdot a$ for some $a \in A_{b} \subseteq A$. Therefore $y=b \cdot v=w \cdot a$ for some $v \in V_{1}$ and $w \in W_{b}$. This implies that $a^{-1} \cdot b=v^{-1} \cdot b^{-1} \cdot w \cdot b \in V_{1}^{-1} \cdot b^{-1} \cdot W_{b} \cdot b$. We have

$$
y \in b \cdot V_{1}=a \cdot\left(a^{-1} \cdot b\right) \cdot V_{1} \subseteq a \cdot V_{1}^{-1} \cdot\left(b^{-1} \cdot W_{b} \cdot b\right) \cdot V_{1} \subseteq a \cdot V_{1}^{3} \subseteq a \cdot V .
$$

Thus, $y \in a \cdot V$ and $y \in W_{b} \cdot a \subseteq V \cdot a$. This proves the lemma. 
We start considering Problem A. For the sake of generality the induced uniformities on $X^{n}$ for an arbitrary $n \in N^{+}$are considered here. From now on the symbols ${ }^{*} \mathcal{V}, \mathcal{V}^{*}$ and ${ }^{*} \mathcal{V}^{*}$ always denote respectively the left, right and two-sided uniformities of the free topological group $F(X)$ on a given space $X$.

THEOREM 1.6. Both uniformities $\left.{ }^{*} \mathcal{V}\right|_{X^{n}}$ and $\left.\mathcal{V}^{*}\right|_{X^{n}}$ are finer than $\mathcal{U}_{X} \times \ldots \times \mathcal{U}_{X}$ (n times) for every space $X$ and each $n \in N^{+}$.

Proof: Let $V$ be an entourage of the diagonal $\triangle_{n}$ in $X^{2 n}, V \in \mathcal{U}_{X} \times \ldots \times \mathcal{U}_{X}$ ( $n$ times). It suffices to find $W^{l} \in{ }^{*} \mathcal{V}$ and $W^{r} \in \mathcal{V}^{*}$ such that $W^{l} \cap X^{2 n} \subseteq V$ and $W^{r} \cap X^{2 n} \subseteq V$.

By the definition of a uniform product (see [2, Chapter 8] or [6]), one can find an entourage $U$ of the diagonal $\Delta_{1}$ in $X^{2}$ such that $(\bar{x}, \bar{y}) \in V$ for all points $\bar{x}=$ $\left(x_{1}, \ldots, x_{n}\right) \in X^{n}$ and $\bar{y}=\left(y_{1}, \ldots, y_{n}\right) \in X^{n}$ satisfying $\left(x_{i}, y_{i}\right) \in U$ for each $i \leqslant n$. Use Corollary 8.1.11 of [2] to define a continuous pseudometric $d$ on $X$ such that $\{(x, y) \in X: d(x, y)<1\} \subseteq U$. Denote by $\hat{d}$ the Graev extension of $d$ to a continuous invariant pseudometric on $G(X)$ and put $O=\{g \in G(X): \widehat{d}(g, e)<1\}$, where $e$ is the identity of $G(X)$. Then define the sets $W^{l}$ and $W^{r}$ by

$$
\begin{aligned}
& W^{\prime}=\left\{\left(x_{1}, \ldots, x_{n}, y_{1}, \ldots, y_{n}\right) \in X^{2 n}: x_{n}^{-1} \cdot \ldots \cdot x_{1}^{-1} \cdot y_{1} \cdot \ldots \cdot y_{n} \in O\right\} \\
& W^{r}=\left\{\left(x_{1}, \ldots, x_{n}, y_{1}, \ldots, y_{n}\right) \in X^{2 n}: x_{1} \cdot \ldots \cdot x_{n} \cdot y_{n}^{-1} \cdot \ldots \cdot y_{1}^{-1} \in O\right\} .
\end{aligned}
$$

It is clear that $\left.W^{l} \in \in^{*} \mathcal{V}\right|_{X^{n}}$ and $\left.W^{r} \in \mathcal{V}^{*}\right|_{X^{n}}$. We claim that $W^{l} \subseteq V$ and $W^{r} \subseteq V$. It suffices to verify the first of these inclusions. Assume that $x_{n}^{-1} \cdot \ldots \cdot x_{1}^{-1} \cdot y_{1} \cdot \ldots \cdot y_{n} \in O$ where $x_{i}, y_{i} \in X$ for each $i \leqslant n$. Then we have

$$
1>\widehat{d}\left(x_{n}^{-1} \cdot \ldots \cdot x_{1}^{-1} \cdot y_{1} \cdot \ldots \cdot y_{n}, e\right)=\sum_{i=1}^{n} d\left(x_{i}, y_{i}\right)
$$

which readily follows from the definition of $\hat{d}$ (see $[5,12])$. In particular, $d\left(x_{i}, y_{i}\right)<1$. for each $i \leqslant n$, and the choice of $d$ and $U$ implies that $\left(x_{1}, \ldots, x_{n}, y_{1}, \ldots, y_{n}\right) \in V$. This proves the inclusion $W^{l} \subseteq V$. An analogous argument shows that $W^{r} \subseteq V$.

A compact space admits only one uniformity compatible with the topology of the space. Therefore, $\left.{ }^{*} \mathcal{V}\right|_{X^{n}}=\left.\mathcal{V}^{*}\right|_{X^{n}}=\left.{ }^{*} \mathcal{V}^{*}\right|_{X^{n}}=\mathcal{U}_{X} \times \ldots \times \mathcal{U}_{X}(n$ times) for any compact space $X ; n \in N^{+}$. Theorem 1.8 below generalises this obvious fact. In its proof we shall use one auxiliary result, which follows from [18, Theorem 2].

LEMMA 1.7. Every pseudo- $\omega_{1}$-compact, $C$-embedded subset $Z$ of a space $T$ is $P$-embedded in $T$, that is, every continuous pseudometric on $Z$ extends to a continuous pseudometric on $T$. 
THEOREM 1.8. For a pseudocompact space $X$, the equalities $\left.{ }^{*} \mathcal{V}\right|_{X^{n}}=\left.\mathcal{V}^{*}\right|_{X^{n}}=$ $\left.{ }^{*} \mathcal{V}^{*}\right|_{X^{n}}=\mathcal{U}_{X} \times \ldots \times \mathcal{U}_{X}$ (n times) hold for each $n \in N^{+}$.

ProOF: Let the space $X$ be pseudocompact and $n \in N^{+}$be arbitrary. Since ${ }^{*} \mathcal{V}^{*}$ is finer than ${ }^{*} \mathcal{V}$ and $\mathcal{V}^{*}$ and both uniformities $\left.{ }^{*} \mathcal{V}\right|_{X^{n}},\left.\mathcal{V}^{*}\right|_{X^{n}}$ are finer than $\mathcal{U}_{n}=$ $\mathcal{U}_{X} \times \ldots \times \mathcal{U}_{X}$ ( $n$ times) (Theorem 1.6), it suffices to show that $\left.{ }^{*} \mathcal{V}^{*}\right|_{X^{n}}=\mathcal{U}_{n}$. We shall prove a more general result: the universal uniformity $\mathcal{W}$ of $F(X)$ restricted to $X^{n}$ coincides with $\mathcal{U}_{n}$. Since $\mathcal{W}$ is finer than ${ }^{*} \mathcal{V}^{*}$, it suffices to verify that $\mathcal{U}_{n}$ is finer than $\left.\mathcal{W}\right|_{X^{n}}$.

The uniformity $\mathcal{W}$ is generated by the family of all continuous pseudometrics on $F(X)$. Let $d$ be one of them. It is necessary to verify that the restriction $\varrho=\left.d\right|_{X^{n}}$ of $d$ to the subspace $X^{n}$ of $F(X)$ is uniformly continuous with respect to $\mathcal{U}_{n}$. Since $X$ is pseudocompact, the natural monomorphism of $F(X)$ to $F(\beta X)$ is a homeomorphic embedding by a theorem of Pestov [9], where $\beta X$ is the Cech-Stone compactification of $X$. So, we can identify $F(X)$ with the corresponding subgroup of $F(\beta X)$ generated by the set $X$. Again, since $X$ is pseudocompact, Theorem 3 of [16] implies that $F(X)$ is $C$-embedded into $F(\beta X)$, that is, every continuous real-valued function on $F(X)$ extends to a continuous function on $F(\beta X)$. Furthermore, the group $F(\beta X)$ is $\sigma$-compact, and hence has countable cellularity by Corollary 2 of [14]. Being dense in $F(\beta X)$, the group $F(X)$ has countable cellularity as well. In particular, $F(X)$ is pseudo- $\omega_{1}$-compact. Applying Lemma 1.7, we conclude that $F(X)$ is $P$-embedded in $F(\beta X)$. Let $\tilde{d}$ be a continuous pseudometric on $F(\beta X)$ which extends $d$. The restriction of $\tilde{d}$ to the subspace $Y=\beta X \times \ldots \times \beta X$ ( $n$ times) of $F(\beta X)$ is an extension of the pseudometric $\varrho$. Since every continuous pseudometric on $F(X)$ extends to a continuous pseudometric on $F(\beta X)$, the universal uniformity $\widetilde{\mathcal{W}}$ of $F(\beta X)$ induces the universal uniformity $\mathcal{W}$ on $F(X)$, that is, $\left.\widetilde{\mathcal{W}}\right|_{F(X)}=\mathcal{W}$. In particular, $\left.\widetilde{\mathcal{W}}\right|_{X^{n}}=\left.\mathcal{W}\right|_{X^{n}}$. Denote by $\tilde{\mathcal{U}}_{1}$ the (unique) uniformity of the space $\beta X$ compatible with its topology. Note that $\left.\tilde{\mathcal{U}}_{1}\right|_{X}=\mathcal{U}_{X}$. Obviously, $\widetilde{\mathcal{W}}$ induces the uniformity $\tilde{\mathcal{U}}_{n}=\tilde{\mathcal{U}}_{1} \times \ldots \times \tilde{\mathcal{U}}_{1}(n$ times) on the compact space $Y$, and $\left.\tilde{U}_{n}\right|_{X^{n}}=\mathcal{U}_{n}$. Therefore, the uniform continuity of $\left.\tilde{d}\right|_{Y}$ with respect to $\tilde{U}_{n}$ implies that $\varrho=\left.\tilde{d}\right|_{X^{n}}$ is a uniformly continuous pseudometric with respect to $\mathcal{U}_{n}$. This proves that $\mathcal{U}_{n}$ is finer than $\left.\widetilde{\mathcal{W}}\right|_{X^{n}}=\left.\mathcal{W}\right|_{X^{n}}$.

We generalise the above theorem in the next section by means of more subtle methods.

\section{Solution of Problem B}

The following theorem completely characterises those spaces $X$ satisfying the equality $\left.{ }^{*} \mathcal{V}^{*}\right|_{X^{2}}=\mathcal{U}_{X} \times \mathcal{U}_{X}$. Recall [15] that a subset $X$ of a topological group $G$ is said to be thin in $G$ if for every neighbourhood $U$ of the identity in $G$ there exists a neighbourhood $V$ of the identity such that $x \cdot V \cdot x^{-1} \subseteq U$ for each $x \in X$. 
Theorem 2.1. The following conditions are equivalent for a Tikhonov space $X$ :

(1) $\left.{ }^{*} \mathcal{V}\right|_{X^{2}}=\mathcal{U}_{X} \times \mathcal{U}_{X}$;

(1') $\left.{ }^{*} \mathcal{V}\right|_{X^{n}}=\mathcal{U}_{X} \times \ldots \times \mathcal{U}_{X}$ (n times) for each $n \geqslant 2$;

(2) $\left.\mathcal{V}^{*}\right|_{X^{2}}=\mathcal{U}_{X} \times \mathcal{U}_{X} ;$

(2') $\left.\mathcal{V}^{*}\right|_{X^{n}}=\mathcal{U}_{X} \times \ldots \times \mathcal{U}_{X}$ (n times) for each $n \geqslant 2$;

(3) $\left.{ }^{*} \mathcal{V}^{*}\right|_{X^{2}}=\mathcal{U}_{X} \times \mathcal{U}_{X}$;

(3') $\left.{ }^{*} \mathcal{V}^{*}\right|_{X^{n}}=\mathcal{U}_{X} \times \ldots \times \mathcal{U}_{X}$ (n times) for each $n \geqslant 2$;

(4) $\left.{ }^{*} \mathcal{V}\right|_{X^{2}}=\left.\mathcal{V}^{*}\right|_{X^{2}}$;

(4') $\left.{ }^{*} \mathcal{V}\right|_{X^{n}}=\left.\mathcal{V}^{*}\right|_{X^{n}}$ for each $n \geqslant 2$;

(5) $X$ is thin in $F(X)$;

(6) there exists an infinite cardinal $\tau$ such that $X$ is a $P_{\tau}$-space and pseudo$\tau$-compact.

Proof: Obviously, $\left(1^{\prime}\right)$ implies (1),.., (4') implies (4). By Theorem 1.6, the implications $(3) \Rightarrow(1),(3) \Rightarrow(2)$ and $\left(3^{\prime}\right) \Rightarrow\left(1^{\prime}\right),\left(3^{\prime}\right) \Rightarrow\left(2^{\prime}\right)$ are valid. It is also clear that $(1) \&(2) \Rightarrow(4)$ and $\left(1^{\prime}\right) \&\left(2^{\prime}\right) \Rightarrow\left(4^{\prime}\right)$; so $(3)$ implies (4) and $\left(3^{\prime}\right)$ implies $\left(4^{\prime}\right)$. The equivalence of (5) and (6) follows from [15, Theorem 3].

$(5) \Rightarrow\left(3^{\prime}\right)$. Let $n$ be any positive integer and $O$ be a neighbourhood of the identity in $F(X)$. Put

$$
\begin{aligned}
U_{O}=\left\{\left(x_{1}, \ldots, x_{n}, y_{1}, \ldots y_{n}\right)\right. & \in X^{2 n} \\
& \left.x_{1} \ldots x_{n} \cdot y_{n}^{-1} \ldots y_{1}^{-1} \in O, x_{n}^{-1} \ldots x_{1}^{-1} \cdot y_{1} \ldots y_{n} \in O\right\} .
\end{aligned}
$$

Then $\left.U_{O} \in{ }^{*} \mathcal{V}^{*}\right|_{X^{n}}$ and we have to find $U \in \mathcal{U}_{X}$ such that the uniform product $U^{n}=U \times \ldots \times U$ ( $n$ times) is contained in $U_{O}$. To this end, choose a symmetric neighbourhood $V$ of the identity such that $V^{n} \subseteq O$. Since $X$ is thin in $F(X)$, one can define a decreasing sequence $V=V_{0} \supseteq V_{1} \supseteq \ldots \supseteq V_{n}$ of open neighbourhoods of the identity in $F(X)$ such that $x^{\varepsilon} \cdot V_{i+1} \cdot x^{-\varepsilon} \subseteq V_{i}$ for all $x \in X, i \leqslant n-1$ and $\varepsilon= \pm 1$. Put $U=\left\{(x, y) \in X^{2}: x^{-1} \cdot y \in V_{n}, x \cdot y^{-1} \in V_{n}\right\}$. Then $U \in \mathcal{U}_{X}$ and we claim that $U$ works. Indeed, let $p=\left(x_{1}, \ldots, x_{n}, y_{1}, \ldots, y_{n}\right)$ be any point of $X^{2 n}$ satisfying $\left(x_{i}, y_{i}\right) \in U$ for each $i \leqslant n$, that is, $p \in U^{n}$. For every $i$ with $2 \leqslant i \leqslant n$ put $g_{i}=x_{1} \cdot \ldots \cdot x_{i-1}$. We have

$(*)$

$x_{1} \cdot \ldots \cdot x_{n} \cdot y_{n}^{-1} \cdot \ldots \cdot y_{1}^{-1}=\left(g_{n} \cdot x_{n} \cdot y_{n}^{-1} \cdot g_{n}^{-1}\right) \cdot\left(g_{n-1} \cdot x_{n-1} \cdot y_{n-1}^{-1} \cdot g_{n-1}^{-1}\right) \cdot \ldots \cdot\left(x_{1} \cdot y_{1}^{-1}\right)$.

By the choice of $p$ and the sets $V_{i}$ we also have $x_{1} \cdot y_{1}^{-1} \in V_{n}, g_{2} \cdot x_{2} \cdot y_{2}^{-1} \cdot g_{2}^{-1} \in$ $g_{2} \cdot V_{n} \cdot g_{2}^{-1} \subseteq V_{n-1}, \ldots \ldots, g_{n} \cdot x_{n} \cdot y_{n}^{-1} \cdot g_{n}^{-1} \subseteq g_{n} \cdot V_{n} \cdot g_{n}^{-1} \subseteq V_{1}$. In its turn, (*) implies that

$$
x_{1} \cdot \ldots \cdot x_{n} \cdot y_{n}^{-1} \cdot \ldots \cdot y_{1}^{-1} \in V_{1} \cdot \ldots \cdot V_{n-1} \cdot V_{n} \subseteq V^{n} \subseteq O
$$


An analogous argument shows that $x_{n}^{-1} \cdot \ldots \cdot x_{1}^{-1} \cdot y_{1} \cdot \ldots \cdot y_{n} \in V^{n} \subseteq O$. Thus, the inclusion $U^{n} \subseteq U_{O}$ is proved.

$(2) \Rightarrow(6)$. Let $\mathcal{T}$ be the topology of $X$. Denote by $\tau$ the minimal cardinality of a subfamily $\theta \subseteq \mathcal{T}$ such that $\bigcap^{\theta} \notin \mathcal{T}$. If the space $X$ is a counterexample to the implication, there must be a locally finite family $\gamma=\left\{U_{\alpha}: \alpha<\tau\right\}$ of open sets in $X$ with $|\gamma|=\tau$. We can assume that $\gamma$ is discrete (see Lemma 1 of [11]). The definition of $\tau$ implies that there exist a point $x^{*} \in X$ and a decreasing sequence $\left\{V_{\alpha}: \alpha<\tau\right\}$ of open neighbourhoods of $x^{*}$ such that $x^{*}$ does not belong to the interior of the intersection $\bigcap_{\alpha<\tau} V_{\alpha}$. For every $\alpha<\tau$ pick a point $a_{\alpha} \in U_{\alpha}$. Let continuous functions $f_{\alpha}$ and $g_{\alpha}$ on $X$ with values in $[0,1]$ be such that $f_{\alpha}\left(a_{\alpha}\right)=1, g_{\alpha}\left(x^{*}\right)=1, f_{\alpha}$ vanishes outside of $U_{\alpha}$ and $g_{\alpha}$ vanishes outside of $V_{\alpha}$. Define continuous pseudometrics $d_{1, \alpha}$ and $\varrho_{\alpha}$ on $X$ by

$$
d_{1, \alpha}(x, y)=\left|f_{\alpha}(x)-f_{\alpha}(y)\right| \text { and } \varrho_{\alpha}(x, y)=\left|g_{\alpha}(x)-g_{\alpha}(y)\right| \text { for all } x, y \in X \text {. }
$$

Apply Theorem 2.1 of [17] to obtain a continuous pseudometric $d_{2, \alpha}$ on $X^{2}$ such that $d_{1, \alpha}$ and $d_{2, \alpha}$ are right-concordant in the sense of Definition 1.3 of [17] and $d_{2, \alpha}$ satisfies the condition

$$
d_{2, \alpha}((a, x),(a, y))=f_{\alpha}(a) \cdot \varrho_{\alpha}(x, y) \text { for all } a, x, y \in X .
$$

Put $d_{1}=\sum_{\alpha<\tau} d_{1, \alpha}$ and $d_{2}=\sum_{\alpha<\tau} d_{2, \alpha}$. Clearly, $d_{1}$ and $d_{2}$ are continuous rightconcordant pseudometrics on $X$ and $X^{2}$ respectively. By Theorem 1.4 of [17] there exists a continuous seminorm $N$ on $G(X)$ satisfying the properties

$$
\begin{gathered}
N\left(a \cdot b^{-1}\right)=d_{1}(a, b) \text { for all } a, b \in X \\
N\left(a \cdot x \cdot y^{-1} \cdot a^{-1}\right)=d_{2}((a, x),(a, y)) \text { for all } a, x, y \in X .
\end{gathered}
$$

Put $O=\{g \in G(X): N(g)<1\}$. Then $O$ is open in $G(X)$ and, a fortiori, in $F(X)$. Finally, define an open entourage $U_{O}^{r}$ of the diagonal $\Delta_{2}$ in $X^{4}$ by

$$
U_{O}^{r}=\left\{(x, y, z, t) \in X^{4}: x \cdot y \cdot t^{-1} \cdot z^{-1} \in O\right\} \text {. }
$$

Clearly, $\left.U_{O}^{r} \in \mathcal{V}^{*}\right|_{X^{2}}$, and we claim that for each continuous pseudometric $\varrho$ on $X$ there exist an ordinal $\alpha<\tau$ and a point $x \in X$ such that $\varrho\left(x^{*}, x\right)<1$ and $a_{\alpha} \cdot x^{*} \cdot x^{-1} \cdot a_{\alpha}^{-1} \notin$ $O$, that is, $\left(a_{\alpha}, x^{*}, a_{\alpha}, x\right) \notin U_{O}^{r}$. The latter will obviously contradict (2).

Indeed, let $\varrho$ be a continuous pseudometric on $X$. Since $x^{*} \notin$ Int $\bigcap_{\alpha<r} V_{\alpha}$, one can find an ordinal $\alpha<\tau$ and a point $x \in X \backslash V_{\alpha}$ such that $\varrho\left(x^{*}, x\right)<1$. We have

$$
\begin{aligned}
N\left(a_{\alpha} \cdot x^{*} \cdot x^{-1} \cdot a_{\alpha}^{-1}\right) & \stackrel{(P 2)}{=} \\
& d_{2}\left(\left(a_{\alpha}, x^{*}\right),\left(a_{\alpha}, x\right)\right) \\
& \geqslant d_{2, \alpha}\left(\left(a_{\alpha}, x^{*}\right),\left(a_{\alpha}, x\right)\right) \stackrel{(R P)}{=} f_{\alpha}\left(a_{\alpha}\right) \cdot\left|g_{\alpha}(x)-g_{\alpha}\left(x^{*}\right)\right|=1,
\end{aligned}
$$


because $f_{\alpha}\left(a_{\alpha}\right)=g_{\alpha}\left(x^{*}\right)=1$ and $g_{\alpha}(x)=0$ (for $x \notin V_{\alpha}$ ). Thus, $N\left(a_{\alpha} \cdot x^{*} \cdot x^{-1} \cdot a_{\alpha}^{-1}\right) \geqslant$ 1 , and hence $a_{\alpha} \cdot x^{*} \cdot x^{-1} \cdot a_{\alpha}^{-1} \notin O$.

(1) $\Rightarrow(6)$. Use the above argument along with Theorerns 1.5 and 2.3 of [17].

$(4) \Rightarrow(6)$. This is the last implication to be proved. However, it has almost been shown in the proof of the implication $(2) \Rightarrow(6)$. Indeed, if $X$ does not satisfy (6), define a continuous seminorm $N$ on $G(X)$ and an open neighbourhood $O$ of the identity in $F(X)$ as above. It was shown that for every continuous pseudometric $\varrho$ on $X$ there exist an ordinal $\alpha<\tau$ and a point $x \in X$ such that $\varrho\left(x^{*}, x\right)<1$ and $a_{\alpha} \cdot x^{*} \cdot x^{-1} \cdot a_{\alpha}^{-1} \notin O$. We claim that for each neighbourhood $W$ of the identity in $F(X)$, the set $U_{W}^{l} \backslash U_{O}^{r}$ is not empty, where $U_{W}^{l}=\left\{(x, y, z, t) \in X^{4}: y^{-1} \cdot x^{-1} \cdot z \cdot t \in W\right\}$. To see this, for a given neighbourhood $W$ of the identity choose a continuous pseudometric $\varrho$ on $X$ such that $\varrho(x, y)<1$ implies $x^{-1} \cdot y \in W$ for all $x, y \in X$. One can find $\alpha<\tau$ and $x \in X$ satisfying the conditions $\varrho\left(x^{*}, x\right)<1$ and $a_{\alpha} \cdot x^{*} \cdot x^{-1} \cdot a_{\alpha}^{-1} \notin O$. We have $\left(x^{*}\right)^{-1} \cdot a_{\alpha}^{-1} \cdot a_{\alpha} \cdot x=\left(x^{*}\right)^{-1} \cdot x \in W$, for $\varrho\left(x^{*}, x\right)<1$. Thus, $\left(a_{\alpha}, x^{*}, a_{\alpha}, x\right) \in U_{W}^{l} \backslash U_{O}^{r}$, which proves our claim. However, this contradicts (4). The theorem is completely proved.

REMARK 2.2. Theorem 2.1 remains valid if one replaces "each" by "some" in conditions $\left(1^{\prime}\right)-\left(4^{\prime}\right)$.

\section{SOlution of Problem $\mathrm{C}$ For ${ }^{*} \mathcal{V}$ and $\mathcal{V}^{*}$}

The following theorem is an almost complete solution (modulo Question 3.2 below) of Problem $\mathrm{C}$ in the case of the left and right group uniformities ${ }^{*} \mathcal{V}$ and $\mathcal{V}^{*}$ of $F(X)$.

THEOREM 3.1. The following implications are valid for every space $X$ :

$(1) \Leftrightarrow(2) \Leftrightarrow(3) \Rightarrow(4) \Rightarrow(5),(1) \Rightarrow(6)$ and $(5) \&(6) \Rightarrow(1)$, where

(1) $\left.{ }^{*} \mathcal{V}\right|_{X^{2}}=\mathcal{U}_{X^{2}}$;

(2) $\left.\mathcal{V}^{*}\right|_{X^{2}}=\mathcal{U}_{X^{2}}$

(3) $\mathcal{U}_{X^{2}}=\mathcal{U}_{X} \times \mathcal{U}_{X}$;

(4) there exists an infinite cardinal $\tau$ such that $X$ is a $P_{\tau}$-space and $X^{2}$ is pseudo- $\tau$-compact;

(5) the projection $\pi: X \times X \rightarrow X$ is $z$-closed (that is, $\pi$ takes zero-sets in $X^{2}$ to closed sets in $X$ );

(6) for every disjoint open cover $\gamma$ of $X^{2}$ there exists a disjoint open cover $\mu$ of $X$ such that $\mu \times \mu=\{U \times V: U, V \in \mu\}$ is finer than $\gamma$.

Proof: $(3) \Rightarrow(1)$. Obviously, $\mathcal{U}_{X^{2}}$ is finer than $\left.{ }^{*} \mathcal{V}\right|_{X^{2}}$. From Theorem 1.6 it follows that $\left.{ }^{*} \mathcal{V}\right|_{X^{2}}$ is finer than $\mathcal{U}_{X} \times \mathcal{U}_{X}$. This proves the implication.

(3) $\Rightarrow(2)$. Just replace ${ }^{*} \mathcal{V}$ by $\mathcal{V}^{*}$ in the above two lines. 
(1) $\Rightarrow(4)$. Suppose that (1) holds. We claim that if there exists a locally finite family of open sets in $X^{2}$ of cardinality $\tau^{+}$for some $\tau$, then the same is true for $X$. Indeed, if $X^{2}$ contains such a family, then the uniform space $\left(X^{2}, \mathcal{U}_{X^{2}}\right)$ is not $\tau$-bounded (Assertion 1.2), and by (1), $\left(X^{2},\left.{ }^{*} \mathcal{V}\right|_{X^{2}}\right)$ is not $\tau$-bounded either. However, * $\mathcal{V}$ induces the maximal uniformity $\mathcal{U}_{X}$ on $X$; hence by Assertion 1.4, $\left(X, \mathcal{U}_{X}\right)$ fails to be $\tau$-bounded. By Assertion 1.2, this means that $X$ contains a locally finite family $\mu$ of open sets with $|\mu|>\tau$.

Now suppose that (4) does not hold. Let $\mathcal{T}$ be the topology of $X$. Denote by $\tau$ the minimal cardinality of a subfamily $\gamma$ of $\mathcal{T}$ such that $\bigcap \gamma$ is not open in $X$, and choose a family $\gamma \subseteq \mathcal{T}$ with a non-open intersection satisfying $|\gamma|=\tau$. Pick a point $a \in \bigcap \gamma \backslash \operatorname{Int}(\bigcap \gamma)$. Since (4) fails, $X^{2}$ contains a locally finite family of open sets of cardinality $\tau$. The above assertion gives us a locally finite family $\mu$ of open sets in $X$ of the same cardinality $\tau$. By Lemma 1 of [11] the family $\mu$ can even be chosen discrete. Let $\mu=\left\{U_{\alpha}: \alpha<\tau\right\}$ and $\gamma=\left\{V_{\alpha}: \alpha<\tau\right\}$. For each $\alpha<\tau$ define continuous real-valued functions $f_{\alpha}$ and $g_{\alpha}$ on $X$ with values in $[0,1]$ such that $f_{\alpha}$ is equal to one at some point of $U_{\alpha}$ and vanishes outside of $U_{\alpha}, g_{\alpha}(a)=1$ and $g_{\alpha}$ vanishes outside $V_{\alpha}$. For any points $x, y, z, t \in X$ put

$$
d((x, y),(z, t))=\sum_{\alpha<\tau}\left|f_{\alpha}(x) \cdot g_{\alpha}(y)-f_{\alpha}(z) \cdot g_{\alpha}(t)\right| .
$$

Since the family $\left\{U_{\alpha} \times V_{\alpha}: \alpha<\tau\right\}$ is discrete in $X^{2}, d$ is a continuous pseudometric on $X^{2}$. Obviously, $d \leqslant 1$. Define an entourage $W$ of the diagonal in $X^{4}$ by $W=$ $\left\{(x, y, z, t) \in X^{4}: d((x, y),(z, t))<1\right\}$. Then $W \in \mathcal{U}_{X^{2}}$. We claim that the existence of $W$ contradicts (1).

Let $O$ be any open neighbourhood of the identity in $F(X)$. Put $V(a)=X \cap a \cdot O$; $V(a)$ is an open neighbourhood of $a$ in $X$. Since $a \notin \operatorname{Int} \bigcap \gamma$, one can find an ordinal $\alpha<\tau$ such that $V(a) \backslash V_{\alpha} \neq \emptyset$. Pick points $b \in V(a) \backslash V_{\alpha}$ and $x \in U_{\alpha}$ with $f_{\alpha}(x)=1$. We have

$$
d((x, a),(x, b)) \geqslant\left|f_{\alpha}(x) \cdot g_{\alpha}(a)-f_{\alpha}(x) \cdot g_{\alpha}(b)\right|=\left|g_{\alpha}(a)-g_{\alpha}(b)\right|=1,
$$

because $b \notin V_{\alpha}$ and $g_{\alpha}(b)=0$. So, $(x, a, x, b) \notin W$. On the other hand, $(x \cdot a)^{-1}$. $(x \cdot b)=a^{-1} \cdot b \in O$, for $b \in V(a) \subseteq a \cdot O$. Thus, we have proved that $W_{O} \backslash W \neq \emptyset$ for every neighbourhood $O$ of the identity in $F(X)$, where $W_{O}=\left\{(x, y, z, t) \in X^{4}\right.$ : $\left.y^{-1} \cdot x^{-1} \cdot z \cdot t \in O\right\}$. This contradicts (1).

$(2) \Rightarrow(4)$. Apply the above reasoning to the pseudometric $\varrho$ on $X^{2}$ defined by $\varrho((x, y),(z, t))=d((y, x),(t, z))$.

(1) $\Rightarrow(3)$. Let (1) hold. Since (1) implies (4), there exists an infinite cardinal $\tau$ such that $X$ is a $P_{\tau}$-space and $X^{2}$ (and, a fortiori, $X$ ) is pseudo- $\tau$-compact. In its 
turn, this and Theorem 2.1 together imply that $\left.{ }^{*} \mathcal{V}\right|_{X^{2}}=\mathcal{U}_{X} \times \mathcal{U}_{X}$. This equality and (1) imply that $\mathcal{U}_{X^{2}}=\mathcal{U}_{X} \times \mathcal{U}_{X}$.

(2) $\Rightarrow(3)$. Just repeat the above argument with $\mathcal{V}^{*}$ instead of ${ }^{*} \mathcal{V}$ and apply (2) instead of (1).

Thus, we have now proved the equivalence of (1), (2) and (3).

(4) $\Rightarrow(5)$. We use the argument from [1]. Let $Z$ be a zero-set in $X \times X$ and $f: X \times X \rightarrow[0,1]$ a continuous function such that $Z=f^{-1}(0)$. Assume that $\pi(Z)$ is not closed in $X$ and choose a point $a \in \operatorname{cl} \pi(Z) \backslash \pi(Z)$, where $\pi$ is the projection of $X \times X$ onto the first factor. We can assume that $f(a, x)=1$ for each $x \in X$-otherwise consider continuous function $g$ on $X \times X$ defined by $g(x, y)=\min \{f(x, y) / f(a, y), 1\}$. Since $X$ is a $P_{\tau}$-space, we can define by induction a sequence $\left\{\left(x_{\alpha}, y_{\alpha}\right): \alpha<\tau\right\}$ of points of $Z$ and two sequences $\left\{W_{\alpha}: \alpha<\tau\right\}$ and $\left\{W_{\alpha}^{\prime}: \alpha<\tau\right\}$ of open subsets of $X \times X$ such that $W_{\alpha}=U_{\alpha} \times V_{\alpha}$ is a neighbourhood of $\left(x_{\alpha}, y_{\alpha}\right)$ satisfying $f\left(W_{\alpha}\right) \subseteq$ $[0,1 / 3], W_{\alpha}^{\prime}=U_{\alpha}^{\prime} \times V_{\alpha}$ is a neighbourhood of $\left(a, y_{\alpha}\right)$ satisfying $f\left(W_{\alpha}^{\prime}\right) \subseteq[2 / 3,1]$, and $\operatorname{cl} U_{\alpha} \subseteq U_{\beta}, U_{\alpha+1} \cup U_{\alpha+1}^{\prime} \subseteq U_{\alpha}$ whenever $\beta<\alpha<\tau$. It is easy to verify that the family $\left\{W_{\alpha}: \alpha<\tau\right\}$ of open sets in $X \times X$ is locally finite, contradicting the pseudo- $\tau$-compactness of $X \times X$.

$(1) \Rightarrow(6)$. It suffices to show that (3) implies (6). Let (3) hold. Consider two cases.

(a) $X$ is not a $P$-space. Since (3) implies (4), $X^{2}$ is pseudocompact. By a theorem of Glicksberg [4], $\beta\left(X^{2}\right) \cong \beta X \times \beta X$. Hence, to prove (6), one can assume that $X$ is compact. To this end, use the well-known facts that a quasicomponent of any point in a compact space coincides with its component (see Theorem 6.1 .23 of [2]) and that a product of two connected sets is connected.

(b) $X$ is a $P$-space. Let $\gamma$ be a disjoint open cover of $X^{2}$. Define a continuous pseudometric $d$ on $X^{2}$ by $d(a, b)=0$ if both $a$ and $b$ lie in the same element of $\gamma$ and $d(a, b)=1$ otherwise. By (3), there exists a continuous pseudometric $\varrho$ on $X$ such that the conditions $\varrho(x, z)<1$ and $\varrho(y, t)<1$ imply $d((x, y),(z, t))<1$ for all $x, y, z, t \in X$. Consider the equivalence relation $\sim$ on $X$ defined by $x \sim y$ if and only if $\varrho(x, y)=0$. The relation $\sim$ defines a partition $\mu$ of $X$ to disjoint open sets, because $X$ is a $P$-space. The definition of $\varrho$ implies that the cover $\{U \times V: U, V \in \mu\}$ refines $\gamma$.

$(5) \&(6) \Rightarrow(1)$. Assume that both (5) and (6) hold and deduce (3). Consider two cases.

(a) $X$ is not a $P$-space. Then (5) implies that $X$ is pseudocompact (otherwise one can define a zero-set in $X \times X$ with a non-closed projection). By a theorem of Tamano (see Problem 3.12.20(b) of [2]), pseudocompactness of $X$ and (5) together imply that $X^{2}$ is pseudocompact. Therefore, $\beta\left(X^{2}\right) \cong \beta X \times \beta X$, which gives us (3). 
(b) $X$ is a $P$-space. Then (6) implies (3). Indeed, let $d$ be any continuous pseudometric on $X^{2}$ and $\sim$ be an equivalence relation on $X^{2}$ defined by $a \sim b$ if and only if $d(a, b)=0$. Since $X$ and $X^{2}$ are $P$-spaces, the relation $\sim$ defines a partition $\gamma$ of $X^{2}$ into open subsets. By (6), there exists a disjoint open cover $\mu$ of $X$ such that $\{U \times V: U, V \in \mu\}$ refines $\gamma$. Define a continuous pseudometric $\varrho$ on $X$ by $\varrho(x, y)=0$ if both $x$ and $y$ lie in some element of $\mu$, and $\varrho(x, y)=1$ otherwise. It is clear that $\varrho(x, z)<1$ and $\varrho(y, t)<1$ imply $d((x, y),(z, t))=0$ for all $x, y, z, t \in X$. This proves $(3)$, and hence (1).

Question 3.2. Does the condition (4) of Theorem 3.1 imply (1)?

\section{Treating Problem $C$}

We prove that every $k_{\omega}$-space $X$ satisfies the equality $\left.{ }^{*} \mathcal{V}^{*}\right|_{X^{2}}=\mathcal{U}_{X^{2}}$ and characterise metrisable spaces satisfying this equality. However, we still have not a complete solution of Problem $\mathrm{C}$. The case of a $k_{\omega}$-space $X$ is considered first. Recall that $X$ is a $k_{\omega}$-space (see $\left.[3,7]\right)$ if there exists a countable increasing sequence $\left\{K_{n}: n \in N\right\}$ of compact sets in $X$ such that $X=\bigcup_{n \in N} K_{n}$ and a subset $F$ of $X$ is closed if and only if $F \cap K_{n}$ is closed for each $n \in N^{+}$.

Denote by $C_{b}(X)$ the linear space of all continuous real-valued bounded functions on $X$ with the sup-norm defined by $\|f\|=\sup _{x \in X}|f(x)|$.

LEMMA 4.1. Let $X$ be a $k_{\omega}$-space and $U$ an open neighbourhood of the diagonal $\triangle_{2}$ in $X^{4}, \triangle_{2}=\left\{(x, y, x, y) \in X^{4}: x, y \in X\right\}$. Then there exists a continuous mapping $f: X \rightarrow C_{b}(X)$ such that for any $x, y, z, t \in X$ the inequalities

$$
\begin{aligned}
& \|f(x)\| \cdot\|f(y)-f(t)\|<1,\|f(z)\| \cdot\|f(y)-f(t)\|<1 \\
& \|f(y)\| \cdot\|f(x)-f(z)\|<1,\|f(t)\| \cdot\|f(x)-f(z)\|<1
\end{aligned}
$$

imply that $(x, y, z, t) \in U$.

Proof: There exists an increasing sequence $\left\{X_{n}: n \in N\right\}$ of compact subsets of $X=\bigcup_{n \in N} X_{n}$ which determines the topology of $X$ according to the definition of a $k_{\omega}$-space. Being $\sigma$-compact and, a fortiori, paracompact, the space $X^{2}$ admits a continuous pseudometric $d$ such that $\left\{(x, y, z, t) \in X^{4}: d((x, y),(z, t))<1\right\} \subseteq U$. (Use Corollary 8.1.11 of [2].) Let $\mathcal{M}$ be the family of all continuous mappings of $X$ onto second-countable spaces. The family $\mathcal{M}$ is $\kappa_{0}$-complete, that is, a diagonal product of any countable subfamily of $\mathcal{M}$ (considered as a mapping onto its image) belongs to $\mathcal{M}$. Put $\mathcal{N}=\left\{\varphi^{4}: \varphi \in \mathcal{M}\right\}$. Then the family $\mathcal{N}$ is $\aleph_{0}$-complete and generates the topology of $X^{4}$. Since $X^{4}$ is $\sigma$-compact, and hence Lindelöf, Corollary 1 of [13] implies that 
$\mathcal{N}$ has the factorisation property, that is, for every continuous mapping $p: X^{4} \rightarrow \mathbf{R}$ one can find $\psi \in \mathcal{N}$ and a continuous mapping $q: \psi\left(X^{4}\right) \rightarrow \mathbf{R}$ such that $p=q \circ \psi$. In particular, there exist $\varphi \in \mathcal{M}$ and a continuous pseudometric $d_{1}$ on $\varphi(X) \times \varphi(X)$ such that $d((x, y),(z, t))=d_{1}((\varphi(x), \varphi(y)),(\varphi(z), \varphi(t)))$ for all $x, y, z, t \in X$. Denote $Y=\varphi(X)$. Let $\varrho_{0}$ be a metric on $Y$ generating the topology of $Y$. Of course, we can assume that $|Y|>1$. One can find $a, b \in Y$ and an integer $k$ such that $k \cdot \varrho_{0}(a, b)>2$. Put $\varrho=\min \left\{1, k \varrho_{0}\right\}$. Then $\varrho$ is a continuous pseudometric on $Y$ with the following property:

(*) for any point $x \in Y$ there exists $y \in Y$ such that $\varrho(x, y)=1$.

The function $\varrho_{2}$ defined by $\varrho_{2}((x, y),(z, t))=\varrho(x, z)+\varrho(y, t)$ for all $x, y, z, t \in Y$ is a continuous metric on $Y^{2}$. For every point $(x, y) \in Y^{2}$ denote

$$
B(x, y)=\left\{(z, t) \in Y^{2}: d_{1}((x, y),(z, t))<1\right\} \text { and } \varepsilon(x, y)=\varrho_{2}\left((x, y), Y^{2} \backslash B(x, y)\right) .
$$

Obviously, $\varepsilon(x, y)>0$ for all $x, y \in Y$. Since $Y_{n}=\varphi\left(X_{n}\right)$ is compact, for every $n \in \mathbb{N}$ there exists $\varepsilon_{n}>0$ such that $\varrho_{2}(\bar{x}, \bar{y})<\varepsilon_{n}$ implies $d_{1}(\bar{x}, \bar{y})<1$ for all $\bar{x}, \bar{y} \in$ $Y_{n}^{2}$. Denote $V_{n}=\left\{(x, y) \in Y^{2}: \varrho_{2}\left((x, y), Y_{n}^{2}\right)<\varepsilon_{n} / 2\right\}$, an open neighbourhood of $Y_{n}^{2}$ in $Y^{2}$. By compactness of $Y_{n}$, there exists an open subset $O_{n}$ of $Y$ such that $Y_{n} \subseteq O_{n}$ and $O_{n} \times O_{n} \subseteq V_{n}$. For every $n \in N$ define a continuous real-valued function $h_{n}$ on $Y$ such that $h_{n}\left(Y_{n}\right)=0, h_{n}\left(Y \backslash O_{n}\right)=4 / \varepsilon_{n+1}$ and $h_{n} \geqslant 0$. Then put $h=\sum_{n=1}^{\infty} h_{n}$, where $h_{0} \equiv 4+4 / \varepsilon_{0}$. Obviously, $4<h(y)<\infty$ for each $y \in Y$, but $h$ can be discontinuous. We claim that the function $\tilde{h}=h \circ \varphi$ on $X$ is continuous. This readily follows from the fact that $X$ is a $k_{\omega}$-space with the decomposition $X=\bigcup_{n=0}^{\infty} X_{n}$ and the choice of the functions $h_{n} ; n \in N$. Put $\tilde{\varrho}(x, y)=\varrho(\varphi(x), \varphi(y))$ for $x, y \in X$. Clearly, $\tilde{\varrho}$ is a bounded continuous pseudometric on $X$.

Consider the mapping $f: X \rightarrow C_{b}(X)$ defined by $[f(x)](y)=\tilde{h}(x) \cdot \widetilde{\varrho}(x, y) ; x, y \in$ $X$. Obviously, $\|f(x)\| \leqslant \widetilde{h}(x)$, because $\tilde{\varrho} \leqslant 1$. On the other hand, $(*)$ implies that there exists a point $y \in Y$ such that $\varrho(x, y)=1$; hence $\|f(x)\|=\widetilde{h}(x)$ for each $x \in X$. Let us check the continuity of $f$. For any $x_{1}, x_{2}, y \in X$ we have

$$
\begin{aligned}
\left|\tilde{h}\left(x_{1}\right) \tilde{\varrho}\left(x_{1}, y\right)-\tilde{h}\left(x_{2}\right) \tilde{\varrho}\left(x_{2}, y\right)\right| \leqslant\left|\widetilde{h}\left(x_{1}\right) \tilde{\varrho}\left(x_{1}, y\right)-\tilde{h}\left(x_{1}\right) \tilde{\varrho}\left(x_{2}, y\right)\right| \\
+\left|\tilde{h}\left(x_{1}\right) \tilde{\varrho}\left(x_{2}, y\right)-\tilde{h}\left(x_{2}\right) \tilde{\varrho}\left(x_{2}, y\right)\right| \leqslant \widetilde{h}\left(x_{1}\right) \widetilde{\varrho}\left(x_{1}, x_{2}\right)+\left|\tilde{h}\left(x_{1}\right)-\tilde{h}\left(x_{2}\right)\right| .
\end{aligned}
$$

Taking the supremum over $y \in Y$ in both left and right parts of the above inequalities, we get $\left\|f\left(x_{1}\right)-f\left(x_{2}\right)\right\| \leqslant \widetilde{h}\left(x_{1}\right) \cdot \varrho\left(x_{1}, x_{2}\right)+\left|\widetilde{h}\left(x_{1}\right)-\widetilde{h}\left(x_{2}\right)\right|$. The latter inequality implies the continuity of $f$.

We claim that the mapping $f$ is as required. To this end, one auxiliary assertion will be useful. 
Claim. $\|f(a)-f(b)\| \geqslant \tilde{\varrho}(a, b)$ for all $a, b \in X$. Moreover, if $\varphi(a) \notin O_{n}$ for some $n \in N$ then $\|f(a)-f(b)\| \geqslant \tilde{\varrho}(a, b) / \varepsilon_{n+1}$.

Indeed, by the definition of the sup-norm and the function $f$ we have $\|f(a)-f(b)\|$ $\geqslant|\tilde{h}(a) \tilde{\varrho}(a, y)-\tilde{h}(b) \tilde{\varrho}(b, y)|$ for each $y \in Y$. Putting $y=b$ in the above inequality, we get $\|f(a)-f(b)\| \geqslant \widetilde{h}(a) \tilde{\varrho}(a, b) \geqslant \tilde{\varrho}(a, b)$, because $\tilde{h}(a)=h(\varphi(a)) \geqslant 1$ for each $a \in X$. Furthermore, if $\varphi(a) \notin O_{n}$ then the definition of the function $h$ implies $h(\varphi(a)) \geqslant 1 / \varepsilon_{n+1}$, and hence $\|f(a)-f(b)\| \geqslant \tilde{h}(a) \tilde{\varrho}(a, b) \geqslant \tilde{\varrho}(a, b) / \varepsilon_{n+1}$. This proves the claim.

Let $x, y, z, t$ be arbitrary points of $X$ satisfying (IL) and (IR). Denote $x^{\prime}=$ $\varphi(x), \ldots, t^{\prime}=\varphi(t)$. First, we show that $d_{1}\left(\left(x^{\prime}, y^{\prime}\right),\left(z^{\prime}, t^{\prime}\right)\right)<1$, or equivalently, $d((x, y),(z, t))<1$. Consider two cases.

CASE 1. $\left\{x^{\prime}, y^{\prime}, z^{\prime}, t^{\prime}\right\} \subseteq O_{0}$. By the definition of $V_{0}$ and $O_{0}$, we have $\varepsilon\left(x^{\prime}, y^{\prime}\right)>\varepsilon_{0} / 2$. By the definition of $\varepsilon\left(x^{\prime}, y^{\prime}\right)$, the latter inequality means that $d_{1}\left(\left(x^{\prime}, y^{\prime}\right),\left(z^{\prime}, t^{\prime}\right)\right)<1$ whenever the point $\left(z^{\prime}, t^{\prime}\right) \in Y^{2}$ satisfies the condition $\varrho_{2}\left(\left(x^{\prime}, y^{\prime}\right),\left(z^{\prime}, t^{\prime}\right)\right) \leqslant \varepsilon_{0} / 2$. So, it suffices to check the inequality $g_{2}\left(\left(x^{\prime}, y^{\prime}\right),\left(z^{\prime}, t^{\prime}\right)\right) \leqslant \varepsilon_{0} / 2$. Using (IL), (IR) and the Claim, we have

$$
\varrho\left(x^{\prime}, z^{\prime}\right)=\widetilde{\varrho}(x, z) \leqslant\|f(x)-f(z)\| \leqslant 1 /\|f(y)\|=1 / \widetilde{h}(y) \leqslant \varepsilon_{0} / 4,
$$

because $\widetilde{h}=\varphi \circ h$ and $h \geqslant 4 / \varepsilon_{0}$. The same argument shows that $\varrho\left(y^{\prime}, t^{\prime}\right) \leqslant \varepsilon_{0} / 4$. Thus, $\varrho_{2}\left(\left(x^{\prime}, y^{\prime}\right),\left(z^{\prime}, t^{\prime}\right)\right)=\varrho\left(x^{\prime}, z^{\prime}\right)+\varrho\left(y^{\prime}, t^{\prime}\right) \leqslant \varepsilon_{0} / 2$, which implies the inequality $d_{1}\left(\left(x^{\prime}, y^{\prime}\right),\left(z^{\prime}, t^{\prime}\right)\right)<1$.

CASE 2. $\left\{x^{\prime}, y^{\prime}, z^{\prime}, t^{\prime}\right\} \backslash O_{0} \neq 0$. Let $n$ be the maximal integer with the property $\left\{x^{\prime}, y^{\prime}, z^{\prime}, t^{\prime}\right\} \backslash O_{n} \neq \emptyset$. Without loss of generality we can assume that $x^{\prime} \notin O_{n}$. From the definition of $n$ it follows that $\left(x^{\prime}, y^{\prime}\right) \in O_{n+1} \times O_{n+1} \subseteq V_{n+1}$, and hence $\varepsilon\left(x^{\prime}, y^{\prime}\right)>$ $\varepsilon_{n+1} / 2$. The definition of $h$ and the fact that $x^{\prime} \notin O_{n}$ imply $h\left(x^{\prime}\right) \geqslant 4 / \varepsilon_{n+1}$. Apply Claim and (IL), (IR) to get the following estimate:

$$
\varrho\left(y^{\prime}, t^{\prime}\right)=\widetilde{\varrho}(y, t) \leqslant\|f(y)-f(t)\| \leqslant 1 /\|f(x)\|=1 / h\left(x^{\prime}\right) \leqslant \varepsilon_{n+1} / 4 .
$$

Then apply the " $\varphi(a) \notin O_{n}$ " part of the Claim and (IL), (IR) to the points $x, y, z, t$ :

$$
\varrho\left(x^{\prime}, z^{\prime}\right)=\tilde{\varrho}(x, z) \leqslant \varepsilon_{n+1} \cdot\|f(x)-f(z)\| \leqslant \varepsilon_{n+1} /\|f(y)\| \leqslant \varepsilon_{n+1} / 4
$$

because $\|f(y)\|=h\left(y^{\prime}\right)$ and $h \geqslant 4$. This implies the estimate

$$
\varrho_{2}\left(\left(x^{\prime}, y^{\prime}\right),\left(z^{\prime}, t^{\prime}\right)\right)=\varrho\left(x^{\prime}, z^{\prime}\right)+\varrho\left(y^{\prime}, t^{\prime}\right)<\varepsilon_{n+1} / 4+\varepsilon_{n+1} / 4=\varepsilon_{n+1} / 2 .
$$

Since $\varepsilon\left(x^{\prime}, y^{\prime}\right)>\varepsilon_{n+1} / 2$, the above inequality implies that $d_{1}\left(\left(x^{\prime}, y^{\prime}\right),\left(z^{\prime}, t^{\prime}\right)\right)<1$. (The argument here is just the same as in Case 1.) 
So, the inequality $d((x, y),(z, t))<1$ is proved. From the definition of the pseudometric $d$ it readily follows that $(x, y, z, t) \in U$. This completes the proof.

THEOREM 4.2. If $X$ is a $k_{\omega}$-space then $\left.{ }^{*} \mathcal{V}^{*}\right|_{X^{2}}=\mathcal{U}_{X^{2}}$.

Proof: Let $U$ be an open entourage of the diagonal $\triangle_{2}$ in $X^{4}$. Define a continuous mapping $f: X \rightarrow C_{b}(X)$ as in Lemma 4.1. Put $g(x)=\|f(x)\|$ and $\varrho(x, y)=\|f(x)-f(y)\|$ for all $x, y \in X$. Then $\varrho_{1}=\min \{\varrho, 1\}$ is a continuous bounded pseudometric on $X$, and we can apply Theorem 2.1 of [17] to the function $f$ and the pseudometric $\varrho_{1}$ to get two continuous seminorms $N_{l}$ and $N_{r}$ on the subgroup $G(X)$ of $F(X)$ satisfying the following conditions for all $x, y, z, t \in X$ :

(1) $N_{l}\left(y^{-1} \cdot x^{-1} \cdot z \cdot y\right)=g(y) \cdot \varrho_{1}(x, z)$ and $N_{r}\left(x \cdot y \cdot z^{-1} \cdot x^{-1}\right)=g(x)$. $\varrho_{1}(y, z)$

(2) $N_{l}\left(y^{-1} \cdot x^{-1} \cdot z \cdot y\right) \leqslant N_{l}\left(y^{-1} \cdot x^{-1} \cdot z \cdot t\right)$ and $N_{r}\left(x \cdot y \cdot t^{-1} \cdot x^{-1}\right) \leqslant$ $N_{r}\left(x \cdot y \cdot t^{-1} \cdot z^{-1}\right)$.

Put $N=N_{l}+N_{r}$ and define an open neighbourhood $O$ of the identity in $F(X)$ by $O=\{g \in G(X): N(g)<1\}$. We claim that the element $V_{O}=\{(g, h) \in F(X) \times F(X)$ : $\left.g^{-1} \cdot h \in O, g \cdot h^{-1} \in O\right\}$ of the uniformity ${ }^{*} \mathcal{V}^{*}$ on $F(X)$ satisfies the condition $V_{O} \cap\left(X^{2} \times X^{2}\right) \subseteq U$. (Recall that we identify $X^{2}$ with the image $i_{2}\left(X^{2}\right) \subseteq F(X)$ under the homeomorphic embedding $i_{2}$, where $i_{2}(x, y)=x \cdot y$.) Indeed, let $x, y, z, t$ be arbitrary points of $X$ and suppose that $((x, y),(z, t)) \in V_{O}$. By the definition of $O$ and $V_{O}$, we have

and

$$
\begin{aligned}
& N_{l}\left(y^{-1} \cdot x^{-1} \cdot z \cdot t\right)=N_{l}\left(t^{-1} \cdot z^{-1} \cdot x \cdot y\right)<1 \\
& N_{r}\left(x \cdot y \cdot t^{-1} \cdot z^{-1}\right)=N_{r}\left(z \cdot t \cdot y^{-1} \cdot x^{-1}\right)<1
\end{aligned}
$$

Use (1) and (2) to conclude that

$$
\begin{array}{ll}
\|f(y)\| \cdot\|f(x)-f(z)\|<1, & \|f(t)\| \cdot\|f(x)-f(z)\|<1, \\
\|f(x)\| \cdot\|f(y)-f(t)\|<1, & \|f(z)\| \cdot\|f(y)-f(t)\|<1 .
\end{array}
$$

These inequalities and the choice of the mapping $f$ together imply that $((x, y),(z, t)) \in$ $U$. Therefore, the inclusion $V_{O} \cap\left(X^{2} \times X^{2}\right) \subseteq U$ is proved.

Theorem 4.4 below gives a solution to Problem $\mathrm{C}$ for metrisable spaces. We start with an auxiliary result.

Let $\gamma$ be a cover of a space $Y$ and $y_{1}, y_{2} \in Y$. We say that $y_{1}$ and $y_{2}$ are $\gamma$ neighbours and write $y_{1} \stackrel{\gamma}{\sim} y_{2}$ if there exists $U \in \gamma$ such that $y_{1}, y_{2} \in U$. Again, $C_{b}(X)$ stands for the linear space of continuous real-valued bounded functions on $X$ with the sup-norm. 
Lemma 4.3. Let $K$ be a compact space with a metric $\varrho_{K}$ and $X$ a locally compact metrisable space. Then for any open covers $\gamma_{1}$ of $X \times K$ and $\gamma_{2}$ of $K \times X$ there exists a continuous mapping $f: X \rightarrow C_{b}(X)$ such that for all $a, b \in K$ and $x \in X$ the inequality $\|f(x)\| \cdot \varrho_{K}(a, b)<1$ implies that $(x, a) \stackrel{\gamma_{1}}{\sim}(x, b)$ and $(a, x) \stackrel{\gamma_{2}}{\sim}(b, x)$.

Proof: Every locally compact metrisable space is a free topological sum of its open $\sigma$-compact subspaces [2, Theorem 5.1.27], so we can assume that $X$ is $\sigma$-compact. Represent $X$ as a union $\bigcup_{i=1}^{\infty} X_{i}$, where $X_{i}$ is open in $X, \operatorname{cl} X_{i} \subseteq X_{i+1}$ and $\operatorname{cl} X_{i}$ is compact for each $i \in N^{+}$. Let $d$ be a metric on $X, d \leqslant 1$. Denote by $i_{0}$ the standard embedding of $X$ to $C_{b}(X),\left[i_{0}(a)\right](x)=d(a, x)$ for all $a, x \in X$. Obviously, we have $\left\|i_{0}(a)-i_{0}(b)\right\|=d(a, b)$ for all $a, b \in X$, so $i_{0}$ is a homeomorphic embedding.

Define metrics $\kappa_{1}$ and $\kappa_{2}$ respectively on $X \times K$ and $K \times X$ by $\kappa_{1}((x, a),(y, b))=$ $d(x, y)+\varrho_{K}(a, b)=\kappa_{2}((a, x),(b, y))$ for all $a, b \in K$ and $x, y \in X$. For every $j \in N^{+}$, let $\varepsilon_{j, 1}$ be a Lebesgue number of the cover $\left\{V \cap\left(X_{j} \times K\right): V \in \gamma_{1}\right\}$ of the compact space $\mathrm{cl} X_{j} \times K$ with respect to $\kappa_{1}$, that is, a positive real number with the property that every pair of points $\bar{x}, \bar{y}$ of $\operatorname{cl} X_{j} \times K$ satisfying $\kappa_{1}(\bar{x}, \bar{y})<\varepsilon_{j, 1}$ is contained in some element of $\gamma_{1}$. Analogously, we define $\varepsilon_{j, 2}$ as a Lebesgue number of the cover $\left\{V \cap\left(K \times \operatorname{cl} X_{j}\right): V \in \gamma_{2}\right\}$ with respect to $\kappa_{2}$.

Denote by $n_{j}$ a positive integer with $1 / n_{j} \leqslant \min \left\{\varepsilon_{j, 1}, \varepsilon_{j, 2}\right\} ; j \in N^{+}$. There exists a continuous mapping $g: X \rightarrow \mathbf{R}$ such that $g(x) \geqslant n_{1}$ for each $x \in X_{1}$ and $g(x) \geqslant n_{j+1}$ for each $x \in X_{j+1} \backslash X_{j}, j \in N^{+}$. Indeed, for every $j \geqslant 2$ we can find a continuous function $g_{j}: X \rightarrow \mathbf{R}$ such that $g_{j}(x)=n_{j+1}$ whenever $x \in \operatorname{cl} X_{j+1} \backslash X_{j}$ and $g_{j}(x)=0$ for each $x \in \operatorname{cl} X_{j-1} \bigcup\left(X \backslash X_{j+2}\right)$. Let also $g_{1}$ be a continuous function such that $g_{1}(x)=\max \left\{n_{1}, n_{2}\right\}$ for all $x \in \operatorname{cl} X_{2}$ and $g_{1}(x)=0$ for each $x \in X \backslash X_{3}$. Then the function $g=\sum_{j=1}^{\infty} g_{j}$ is continuous and has the above property.

Put $f(x)=g(x) \cdot i_{0}(x)$ for every $x \in X$. Obviously, the mapping $f: X \rightarrow$ $C_{b}(X)$ is continuous. Let points $a, b \in K$ and $x \in X$ be arbitrary and suppose that $\|f(x)\| \cdot \varrho_{K}(a, b)<1$. Denote by $j$ the minimal integer with $x \in X_{j}$. Then $\|f(x)\|=$ $|g(x)| \cdot\left\|i_{0}(x)\right\|=g(x) \geqslant n_{j}$. It is clear that $\kappa_{1}((x, a),(x, b))=d(x, x)+\varrho_{K}(a, b)=$ $\varrho_{K}(a, b)$, and hence

$$
1>\|f(x)\| \cdot e_{K}(a, b) \geqslant n_{j} \cdot e_{K}(a, b)=n_{j} \cdot \kappa_{1}((x, a),(x, b)) .
$$

So, the choice of $n_{j}$ and $\varepsilon_{j}$ implies that $(x, a) \stackrel{\gamma_{1}}{\sim}(x, b)$. The same argument shows that $(a, x) \stackrel{\gamma_{2}}{\sim}(b, x)$.

THEOREM 4.4. The following conditions are equivalent for any metrisable space $X$ :

(a) $\left.{ }^{*} \mathcal{V}^{*}\right|_{X^{2}}=\mathcal{U}_{X^{2}}$; 
(b) either $X$ is locally compact or the set $X^{\prime}$ of non-isolated points of $X$ is compact.

Proof: $(a) \Rightarrow(b)$. Assume the contrary. Let $X$ be not locally compact and $X^{\prime}$ be not compact. By our assumption, there exists a point $a \in X$ the closure of any neighbourhood of which is not compact. Using the non-compactness of $X^{\prime}$, we can choose a base $\left\{U_{n}: n \in N^{+}\right\}$of $X$ at $a$ such that $\operatorname{cl} V_{n+1} \subseteq V_{n}, \operatorname{cl} V_{n} \backslash V_{n+1}$ is not compact for each $n \in N^{+}$and $X^{\prime} \backslash V_{1}$ is not compact as well. For every $n \in N^{+}$ choose an infinite closed discrete subset $\left\{x_{n, k}: k \in N^{+}\right\}$of $X$ lying in $V_{n} \backslash V_{n+1}$. Let also $\left\{y_{n}: n \in N^{+}\right\}$be an infinite closed discrete subset of $X^{\prime} \backslash V_{1}$. For every $n \in N^{+}$choose a sequence $\left\{z_{n, l}: l \in N^{+}\right\} \subseteq X \backslash\left(V_{1} \cup\left\{y_{n}\right\}\right)$ converging to $y_{n}$. It is easy to see that the set $F=\left\{\left(x_{n, k}, z_{n, k}, x_{n, k}, y_{n}\right): n, k \in N^{+}\right\}$is closed in $X^{4}$. Let $\triangle_{2}=\{(x, y, x, y): x, y \in X\}$ be the diagonal in $X^{4}$. Obviously, $F$ and $\triangle_{2}$ are disjoint, so $U=X^{4} \backslash F$ is an open neighbourhood of $\triangle_{2}$ in $X^{4}$. The metrisability of $X$ implies that $U \in \mathcal{U}_{X^{2}}$.

Let $O$ be an arbitrary neighbourhood of the identity $e$ in $F(X)$. There exists a neighbourhood $O_{1}$ of $e$ such that $O_{1}^{-1}=O_{1}$ and $O_{1}^{3} \subseteq O$. Choose a neighbourhood $O_{2}$ of $e$ so that $O_{2}^{-1}=O_{2} \subseteq O$ and $a \cdot O_{2} \cdot a^{-1} \subseteq O_{1}$. Put $W=X \cap\left(O_{1} \cdot a\right)$. Since the sets $U_{n}, n \in N^{+}$, form a base of $X$ at $a$ and $W$ is a neighbourhood of the point $a$ in $X$, there exists an integer $p \in N^{+}$such that $x_{n, k} \in W$ for all $n \geqslant p$ and $k \in N^{+}$ (it suffices to choose $p$ with $U_{p} \subseteq W$ ). Choose $l \in N^{+}$so that $z_{p, k} \in O_{2} \cdot y_{p}$ for all $k \geqslant l$. The choice of $l$ implies that

$$
z_{p, l} \cdot y_{p}^{-1} \in O_{2} \text { and } y_{p} \cdot z_{p, l}^{-1} \in O_{2}^{-1}=O_{2}
$$

We have also

(2) $x_{p, l} \cdot\left(z_{p, l} \cdot y_{p}^{-1}\right) \cdot x_{p, l}^{-1} \in\left(O_{1} \cdot a\right) \cdot O_{2} \cdot\left(a^{-1} \cdot O_{1}^{-1}\right)=O_{1} \cdot\left(a \cdot O_{2} \cdot a^{-1}\right) \cdot O_{1} \subseteq O_{1}^{3} \subseteq O$.

Consider the entourage $U_{O}$ of the diagonal in $X^{4}$ generated by $O$,

$$
U_{O}=\left\{(x, y, z, t) \in X^{4}: x \cdot y \cdot t^{-1} \cdot z^{-1} \in O \text { and } y^{-1} \cdot x^{-1} \cdot z \cdot t \in O\right\} .
$$

Then $\left.U_{O} \in{ }^{*} \mathcal{V}^{*}\right|_{X^{2}}$ and from (1), (2) and the inclusion $O_{2} \subseteq O$ it follows that $\left(x_{p, l}, z_{p, l}, x_{p, l}, y_{p}\right) \in U_{O} \cap F$. Thus, we have proved that $U_{O} \backslash U \neq \emptyset$ for each neighbourhood $O$ of the identity in $F(X)$, that is, $\left.{ }^{*} \mathcal{V}^{*}\right|_{X^{2}} \neq \mathcal{U}_{X^{2}}$.

$(b) \Rightarrow(a)$. We consider two cases.

I. $X^{\prime}$ is compact. Let $U$ be an open entourage of the diagonal $\triangle_{2}$ in $X^{4}$. Since $X^{2}$ is paracompact, there exists an open symmetric neighbourhood $U_{1}$ of $\triangle_{2}$ in $X^{4}$ such that $U_{1} \circ U_{1} \subseteq U$. Denote by $\varrho_{0}$ a bounded metric on $X$ that induces the 
topology of $X$. Define another metric $\varrho$ on $X$ by $\varrho(x, y)=0$ if $x=y$ and $\varrho(x, y)=$ $\inf \left\{\varrho_{0}(x, a)+\varrho_{0}(a, y): a \in X^{\prime}\right\}$ otherwise. We omit an easy proof of the fact that $\varrho$ satisfies the inequality of triangle, is continuous bounded and generates the same topology of $X$. From the definition of $\varrho$ it also follows that

$$
\left.\varrho\right|_{X^{\prime}}=\left.\varrho_{0}\right|_{X^{\prime}} \text { and } \varrho(a, b) \geqslant \varrho\left(a, X^{\prime}\right) \text { for all } a, b \in X, a \neq b
$$

Since $X^{\prime}$ is compact, there exists $\varepsilon>0$ such that $(x, y, z, t) \in U_{1}$ whenever $x, y \in X^{\prime}, z, t \in X$ and $\varrho(x, z)<\varepsilon, \varrho(y, t)<\varepsilon$. Put $V=\{x \in X$ : $\left.\varrho\left(x, X^{\prime}\right)<\varepsilon / 2\right\}$. Then $V$ is a clopen neighbourhood of $X^{\prime}$ in $X$. Using the compactness of $X^{\prime}$ once again, for each $x \in X \backslash V$ choose $\varepsilon_{x}>0$ so that all the points $(x, t, x, y),(x, y, x, t),(t, x, y, x),(y, x, t, x)$ belong to $U_{1}$ for all $y \in X^{\prime}$ and $t \in X$ satisfying $\varrho(y, t)<\varepsilon_{x}$.

Denote by $C_{b}(X)$ the space of all continuous real-valued bounded furctions on $X$ with the sup-norm $\|\cdot\|$. Let $h: X \rightarrow \mathrm{R}$ be a continuous function such that $h(y) \geqslant$ $1+2 / \varepsilon$ for all $y \in X$ and $h(x) \geqslant 1+2 / \varepsilon_{x}$ for all $x \in X \backslash V$. Define a mapping $f: X \rightarrow C_{b}(X)$ by $[f(x)](y)=h(x) \cdot \varrho(x, y) ; x, y \in X$. Apply the argument in the proof of Lemma 4.1 to verify that $f$ is continuous and has the following properties:

$$
\begin{gathered}
\|f(x)-f(y)\| \geqslant(1+2 / \varepsilon) \cdot \varrho(x, y) \text { for all } x, y \in X \\
\|f(x)-f(y)\| \geqslant\left(1+2 / \varepsilon_{x}\right) \cdot \varrho(x, y) \text { whenever } x \in X \backslash V \text { and } y \in X .
\end{gathered}
$$

In particular, the inequality $\|f(x)-f(y)\| \geqslant \varrho(x, y)$ holds for all $x, y \in X$.

Put $\varrho_{1}(x, y)=\|f(x)-f(y)\|$ for all $x, y \in X$ and apply Theorems 2.1 and 2.3 of [17] to the metric $\varrho_{1}$ and the function $f$ on $X$ to obtain continuous seminorms $N_{l}$ and $N_{r}$ on $G(X)$ satisfying the following conditions for all $x, y, z, t \in X$ :

(i) $N_{l}\left(y^{-1} \cdot x^{-1} \cdot z \cdot y\right)=\|f(y)\| \cdot \varrho_{1}(x, z)$ and $N_{r}\left(x \cdot y \cdot t^{-1} \cdot x^{-1}\right)=\|f(x)\| \cdot$ $e_{1}(y, t)$

(ii) $N_{l}\left(y^{-1} \cdot x^{-1} \cdot z \cdot t\right) \geqslant \varrho_{1}(x, z)+\varrho_{1}(y, t)$ and $N_{r}\left(x \cdot y \cdot t^{-1} \cdot z^{-1}\right) \geqslant \varrho_{1}(x, z)$ $+\varrho_{1}(y, t)$.

We put $N=N_{l}+N_{r}$ and $O=\{g \in G(X): N(g)<1\}$. Then $O$ is an open neighbourhood of the identity in $F(X)$ and we claim that the entourage $V_{O}=\left\{(x, y, z, t) \in X^{4}: y^{-1} \cdot x^{-1} \cdot z \cdot t \in O, x \cdot y \cdot t^{-1} \cdot z^{-1} \in O\right\}$ of the diagonal $\triangle_{2}$ in $X^{4}$ is contained in $U$. Indeed, if $(x, y, z, t) \in V_{O}$ then $N_{l}\left(y^{-1} \cdot x^{-1} \cdot z \cdot t\right)<1$ and $N_{r}\left(x \cdot y \cdot t^{-1} \cdot z^{-1}\right)<1$. Suppose that $\{x, y, z, t\} \subseteq V$. There exist points $x_{1}, y_{1} \in X^{\prime}$ such that $\varrho\left(x, x_{1}\right)<\varepsilon / 2$ and $\varrho\left(y, y_{1}\right)<\varepsilon / 2$. Applying (4) and (ii), we get

$$
2 / \varepsilon \cdot \varrho(x, z) \leqslant \varrho_{1}(x, z) \leqslant N_{r}\left(x \cdot y \cdot t^{-1} \cdot z^{-1}\right)<1 \text {, that is, } \varrho(x, z)<\varepsilon / 2 .
$$


From the choice of the point $x_{1}$ it follows that $\varrho\left(x, x_{1}\right)<\varepsilon / 2_{\text {; }}$ therefore $\varrho\left(x_{1}, z\right) \leqslant$ $\varrho\left(x_{1}, x\right)+\varrho(x, z)<\varepsilon / 2+\varepsilon / 2=\varepsilon$. Analogously, one can show that $\varrho\left(y_{1}, t\right)<\varepsilon$. These two inequalities and the choice of $\varepsilon$ together imply that $\left(x_{1}, y_{1}, z, t\right) \in U_{1}$. By the same reasoning we also have $\left(x_{1}, y_{1}, x, y\right) \in U_{1}$. It remains to note that $U_{1}$ is symmetric, and hence $(x, y, z, t) \in U_{1} \circ U_{1} \subseteq U$.

We can now assume that $\{x, y, z, t\} \backslash V \neq \emptyset$. It suffices to consider the case $x \notin V$. We claim that $z=x$. Indeed, by (3), $\varrho(x, z) \geqslant \varrho\left(x, X^{\prime}\right) \geqslant \varepsilon x / 2$ if $z \neq x$. So, (ii) and (5) imply that

$$
N_{r}\left(x \cdot y \cdot t^{-1} \cdot z^{-1}\right) \geqslant \varrho_{1}(x, z) \geqslant 2 / \varepsilon_{x} \cdot \varrho(x, z) \geqslant 1,
$$

which contradicts the inequality $N_{r}\left(x \cdot y \cdot t^{-1} \cdot z^{-1}\right)<1$. Thus, $z=x$. Further, by (i) and (5), we have

$$
1>N_{r}\left(x \cdot y \cdot t^{-1} \cdot x^{-1}\right)=\|f(x)\| \cdot \varrho_{1}(y, t) \geqslant 2 / \varepsilon_{x} \cdot \varrho(y, t), \text { that is, } \varrho(y, t)<\varepsilon_{x} / 2 .
$$

We claim that either $y=t$ or $\varrho\left(y, X^{\prime}\right)<\varepsilon_{x} / 2$. Suppose not, let $y \neq t$ and $\varrho\left(y, X^{\prime}\right) \geqslant$ $\varepsilon_{x} / 2$. Then $\varrho(y, t) \geqslant \varrho\left(y, X^{\prime}\right) \geqslant \varepsilon_{x} / 2$ by (3), which contradicts the above inequality. The case $y=t$ is trivial: $(x, y, z, t)=(x, y, x, y) \in \Delta_{2} \subseteq U$; hence we assume that $y \neq t$. There exists a point $y_{1} \in X^{\prime}$ such that $\varrho\left(y, y_{1}\right)<\varepsilon_{x} / 2$, and we have $\varrho\left(y_{1}, t\right) \leqslant$ $\varrho\left(y_{1}, y\right)+\varrho(y, t)<\varepsilon_{x} / 2+\varepsilon_{x} / 2=\varepsilon_{x}$, that is, $\varrho\left(y_{1}, t\right)<\varepsilon_{x}$. Since $y_{1} \in X^{\prime}$, these two inequalities together with the definition of $\varepsilon_{x}$ imply that $\left(x, y, x, y_{1}\right) \in U_{1}$ and $\left(x, y_{1}, x, t\right) \in U_{1}$. Therefore, $(x, y, z, t)=(x, y, x, t) \in U_{1} \circ U_{1} \subseteq U$. This completes the proof of the inclusion $V_{O} \subseteq U$. Since the entourage $U$ of the diagonal $\Delta_{2}$ in $X^{4}$ was chosen arbitrarily and $\left.V_{O} \in{ }^{*} \mathcal{V}^{*}\right|_{X^{2}}$, the equality $\mathcal{U}_{X^{2}}=\left.{ }^{*} \mathcal{V}^{*}\right|_{X^{2}}$ is proved.

II. $X$ is locally compact. Suppose we are given an entourage $U$ of the diagonal $\Delta_{2}$ in $X^{4}, U \in \mathcal{U}_{X^{2}}$. Choose an entourage $V$ of $\Delta_{2}$ in $X^{4}$ with $V \circ V \subseteq U$. There exists an open cover $\gamma$ of $X^{2}$ such that $\bigcup\{A \times A: A \in \gamma\} \subseteq V$. Since $X$ is paracompact, there exists an open locally finite cover $\mu$ of $X$ such that cl $W$ is compact for each $W \in \mu$. Let $\varrho$ be a bounded continuous metric on $X$ such that $\left\{(x, y) \in X^{2}\right.$ : $\varrho(x, y)<1\} \subseteq \bigcup\{W \times W: W \in \mu\}$. By Lemma 4.3, for every $W \in \mu$ there exists a continuous mapping $f_{W}: X \rightarrow C_{b}(X)$ such that for all $a, b \in \mathrm{cl} W$ and $x \in X$ the inequality $\left\|f_{W}(x)\right\| \cdot \varrho(a, b)<1$ implies that $(x, a) \stackrel{\mathcal{\nu}}{\sim}(x, b)$ and $(a, x) \stackrel{\mathcal{\gamma}}{\sim}(b, x)$. Apply Theorem 3.1 of [17] to define a continuous seminorm $N$ on $G(X)$ such that $N(g) \geqslant$ $\widehat{\varrho}(g, e)$ for each $g \in G(X)$ ( $\varrho$ is the Graev extension of $\varrho$ to an invariant pseudometric on $G(X))$ and $N\left(a^{e} \cdot x^{e} \cdot y^{-\varepsilon} \cdot b^{-\varepsilon}\right) \geqslant \max \left\{\left\|f_{W}(a)\right\|,\left\|f_{W}(b)\right\|\right\} \cdot \varrho(x, y)$ whenever the points $a, b, x, y \in X$ satisfy the conditions $N\left(a^{e} \cdot x^{e} \cdot y^{-e} \cdot b^{-e}\right)<1, \varepsilon= \pm 1$ and $x, y \in W \in \mu$. Then $O=\{g \in G(X): N(g)<1\}$ is an open neighbourhood of the identity in $F(X)$, and we claim that the entourage

$$
U_{O}=\left\{(a, x, b, y) \in X^{4}: a \cdot x \cdot y^{-1} \cdot b^{-1} \in O, x^{-1} \cdot a^{-1} \cdot b \cdot y \in O\right\}
$$


of the diagonal $\Delta_{2}$ in $X^{4}$ is contained in $U$. Indeed, let $(a, x, b, y) \in U_{O}$. Then $N\left(a \cdot x \cdot y^{-1} \cdot b^{-1}\right)<1$ and $N\left(x^{-1} \cdot a^{-1} \cdot b \cdot y\right)<1$. Since $N(g) \geqslant \hat{\varrho}(g, e)$ for each $g \in G(X)$, we have

$$
\varrho(a, b)+\varrho(x, y)=\widehat{\varrho}\left(a \cdot x \cdot y^{-1} \cdot b^{-1}, e\right) \leqslant N\left(a \cdot x \cdot y^{-1} \cdot b^{-1}\right)<1 .
$$

In particular, $\varrho(a, b)<1$ and $\varrho(x, y)<1$. The choice of $\varrho$ implies that there exist elements $W, W^{\prime}$ of $\mu$ such that $a, b \in W$ and $x, y \in W^{\prime}$. By the choice of $N$, we have $\left\|f_{W}(a)\right\| \cdot \varrho(x, y) \leqslant N\left(a \cdot x \cdot y^{-1} \cdot b^{-1}\right)<1$ and $\left\|f_{W}(y)\right\| \cdot \varrho(a, b) \leqslant N\left(x^{-1} \cdot a^{-1} \cdot b \cdot y\right)<$ 1. In its turn, the choice of the functions $f_{W}$ and $f_{W^{\prime}}$ implies that $(a, x) \stackrel{\mathcal{\gamma}}{\sim}(a, y)$ and $(a, y) \stackrel{\gamma}{\sim}(b, y)$. Since $\bigcup\{A \times A: A \in \gamma\} \subseteq V$, we conclude that $(a, x, a, y) \in V$ and $(a, y, b, y) \in V$. It remains to note that $V \circ V \subseteq U$, and hence $(a, x, b, y) \in U$. This proves the inclusion $U_{O} \subseteq U$.

\section{REFERENCES}

[1] W.W. Comfort and A.W. Hager, 'The projection mapping and other continuous functions on a product space', Math. Scand. 28 (1971), 77-90.

[2] R. Engelking, General topology (PWN, Warsaw, 1977).

[3] S.P. Franklin and B.V.S. Thomas, 'A survey of $k_{\omega}$-spaces', Topology Proc. 2 (1977), 111-124.

[4] I. Glicksberg, 'Stone-Čech compactifications of products', Trans. Amer. Math. Soc. 90 (1959), 369-382.

[5] M.I. Graev, 'Free topological groups', (in Russian), Izv. Akad. Nauk SSSR 12 (1948), 279-324 (English translation: Translat. Amer. Math. Soc. 8 (1962), 305-364.).

[6] J.R. Isbell, Uniform spaces, Mathematical Surveys 12 (American Mathematical Society, Providence, 1964).

[7] E. Katz, 'Free products in the category of $k_{\omega}$-groups', Pacific J. Math. 59 (1975), 493-495.

[8] E.C. Nummela, 'Uniform free topological groups and Samuel compactifications', Topology Appl. 13 (1982), 77-83.

[9] V.G. Pestov, 'Some properties of free topological groups', (in Russian), Vestnik Moskov. Univ. Ser.I Matem. Mekh. (1982), 35-37 (English translation: Moscow Univ. Math. Bull. 37, 46-49).

[10] W. Roelcke and S. Dierolf, Uniform structures on topological groups and their quotients (New York, 1981).

[11] E.V. Ščepin, 'Real-valued functions and canonical sets in Tikhonov products and topological groups', (in Russian), Uspekhi Mat. Nauk 31 (1976), 17-27 (English translation: Russian Math. Surveys 31 (1976)).

[12] O.V. Sipacheva and V.V. Uspenskii, 'Free topological groups with no small subgroups and Graev metrics', (in Russian), Vestnik Moskov. Univ. Ser. I Mat. Mekh. (1987), 21-24, (English translation: Moscow Univ. Math. Bull. 42, 24-29). 
[13] M.G. Tkačenko, 'Some results on inverse spectra I', Comment. Math. Univ. Carolin. 22 (1981), 621-633.

[14] M.G. Tkačenko, 'The Souslin property in free topological groups on bicompacta', (in Russian), Mat. Zametki 34 (1983), 601-607, (English translation: Mathematical Notes 34, 790-793).

[15] M.G. Tkačenko, 'On some properties of free topological groups', (in Russian), Mat. Zametki 37 (1985), 110-118, (English translation: Mathematical Notes 37 (1985), 62-66).

[16] M.G. Tkačenko, 'Boundedness and pseudocompactness in topological groups', (in Russian), Matem. Zametky 41 (1987), 400-405, (English translation: Mathematical Notes 41, 229-231).

[17] M.G. Tkačenko, 'On group uniformities on square of a space and extending pseudometrics', Bull. Austral. Math. Soc. 51 (1995), 309-335.

[18] V.V. Uspenskiǐ, 'Free topological groups on mertizable spaces', (in Russian), Izv. Acad. Nauk SSSR 54 (1990), 1295-1319.

Departamento de Matemáticas

Universidad Autónoma Metropolitana

Av. Michoacan y La Purísima

Iztapalapa, A.P. 55-532, C.P. 09340

México, D.F.

e-mail: mich@xanum.uam.mx 